\title{
Quantification of a bacterial secondary metabolite by SERS combined with SLM extraction for bioprocess monitoring
}

Morelli, Lidia; Andreasen, Sune Zoëga; Jendresen, Christian Bille; Nielsen, Alex Toftgaard; Emnéus, Jenny; Zor, Kinga; Boisen, Anja

\section{Published in:}

Analyst

Link to article, DOI:

$10.1039 / \mathrm{c} 7 \mathrm{an} 01393 \mathrm{k}$

Publication date:

2017

Document Version

Peer reviewed version

Link back to DTU Orbit

Citation (APA):

Morelli, L., Andreasen, S. Z., Jendresen, C. B., Nielsen, A. T., Emnéus, J., Zor, K., \& Boisen, A. (2017).

Quantification of a bacterial secondary metabolite by SERS combined with SLM extraction for bioprocess monitoring. Analyst, 142(23), 4553-4559 . https://doi.org/10.1039/c7an01393k

\section{General rights}

Copyright and moral rights for the publications made accessible in the public portal are retained by the authors and/or other copyright owners and it is a condition of accessing publications that users recognise and abide by the legal requirements associated with these rights.

- Users may download and print one copy of any publication from the public portal for the purpose of private study or research.

- You may not further distribute the material or use it for any profit-making activity or commercial gain

- You may freely distribute the URL identifying the publication in the public portal 


\section{Quantification of a bacterial secondary metabolite by SERS combined with SLM extraction for bioprocess monitoring}

Received 00th January 20xx, Accepted 00th January 20xx DOI: $10.1039 / \times 0 \times x 00000 x$

www.rsc.org/

\author{
Lidia Morelli, ${ }^{a *}$ Sune Zoëga Andreasen, ${ }^{a}$ Christian Bille Jendresen, ${ }^{\mathrm{b}}$ Alex Toftgaard Nielsen, ${ }^{\mathrm{b}}$ Jenny \\ Emnéus, ${ }^{a}$ Kinga Zór, ${ }^{a}$ and Anja Boisen ${ }^{a}$
}

During the last decades, great advances were reached in high-throughput design and building of genetically engineered microbial strains, leading to a need for fast and reliable screening methods. We developed and optimized a microfluidic supported liquid membrane (SLM) extraction device and combined it with surface enhanced Raman scattering (SERS) sensing for the screening of a biological process, namely for the quantification of a bacterial secondary metabolite, $p$ coumaric acid ( $\mathrm{pHCA}$ ), produced by Escherichia coli. The microfluidic device proved to be robust and reusable, enabling efficient removal of interfering compounds from the real samples, reaching more than 13-fold up-concentration of the donor at $10 \mu \mathrm{L} / \mathrm{min}$ flow rate. With this method we quantified pHCA directly from bacterial supernatant, distinguishing between various culture conditions based on pHCA production yield. The obtained data showed good correlation with HPLC

analysis

\section{Introduction}

Research in the field of metabolic engineering has been significantly growing over the last decades. ${ }^{1}$ In fact, in many cases microbial factories provide a more sustainable production of economically valuable compounds, compared to chemical synthesis or extraction from natural sources. ${ }^{2}$ Modern genetic engineering approaches rely on a design, building and testing cycle. While there have been great advances in the design and building steps, testing typically remains a bottleneck. ${ }^{1,3}$ Therefore, there is an increasing need for fast and reliable screening methods. Current standard techniques, such as high-performance liquid chromatography (HPLC) and gas chromatography/mass spectroscopy are robust, accurate and reliable, but they have several drawbacks, as they need expensive and bulky instrumentation and skilled personnel to optimize the detection protocols. Furthermore, the detection process is time consuming and requires large amounts of solvents. Hence, fast, cost-effective and high-throughput detection would represent a significant improvement and an important innovation in the field. ${ }^{4-6}$

Escherichia coli (E. coli) strains expressing tyrosine ammonia lyase (TAL) are established biological systems for the production of $p$-coumaric acid (pHCA). ${ }^{7}$ The TAL enzymatic reaction is a well-known process in the natural phenols biosynthesis pathway, which transforms tyrosine (Tyr) into pHCA. The resulting phenolic compound has antioxidant and antimicrobial activity and has several commercial applications, for instance in cosmetic $^{8}$ and food industry. ${ }^{9}$ Metabolic engineering of $E$. coli for optimized production of pHCA has many challenges. Besides differences between bacterial strains, production may also vary according to culture conditions, such as medium composition and presence or

\footnotetext{
a. Department of Micro- and Nanotechnology, Technical University of Denmark, 2800 Kgs. Lyngby, Denmark.

b. The Novo Nordisk Foundation Center for Biosustainability, Technical University of Denmark, 2800 Kgs. Lyngby, Denmark.

*e-mail:Imor@nanotech.dtu.dk, phone: +4591734340

Electronic Supplementary Information (ESI) available: Additional material about typical SERS calibration curves at different EtOH dilutions, SERS spectra of Tyr and pHCA in acceptor buffer and results of different rinsing techniques of the SLM microfluidic device in terms of memory effect. See DOI: 10.1039/x0xx00000x
}

absence of nutrients. ${ }^{10}$ Therefore, a time efficient method with potential for high-throughput screening would be extremely beneficial for optimizing metabolic engineering steps. ${ }^{11,12}$

In recent years, surface enhanced Raman scattering (SERS) has proven to be a time-effective and versatile technique, ${ }^{13}$ and a useful analytical tool for quantitative sensing in biological

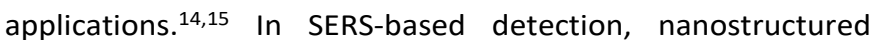
metallic surfaces enhance the Raman signal, enabling detection of analytes at low concentrations with a specific molecular fingerprint. ${ }^{16}$ In order to maximize the enhancement, a molecule should be located within a few nanometers or adsorbed to the active surface, ${ }^{17}$ avoiding excess of salts and interfering compounds, which could dramatically decrease sensitivity. ${ }^{18}$

Bacterial supernatant is a complex solution, containing salts, proteins and metabolites other than the compound of interest. ${ }^{19}$ Furthermore, a certain amount of the substrate needed for production of secondary metabolites can be found at different time points in the supernatant, leading to overlapping spectral features. Therefore, sample pretreatment and cleanup is an important step to reduce the amount of interfering compounds and therefore increase the sensitivity for the compound of interest.

In general, many commonly used separation techniques (e.g. chromatography) are coupled to various detection methods (e.g. spectroscopy) in order to increase sensing accuracy and sensitivity. In spite of their high selectivity, these separation techniques often require large volumes of eluents and complex instrumentation. Other techniques, such as liquidliquid extraction (LLE) and solid phase extraction, are also widely used for extraction of organic analytes from aqueous solutions. ${ }^{20}$

Among membrane-based extraction techniques, supported liquid membrane (SLM) extraction is a powerful and versatile technique, and able to reach high selectivity and enrichment of compounds of interest. ${ }^{21}$ It is based on a small amount of organic solvent immobilized through capillary force in the pores of a porous membrane, placed in between a donor and an acceptor phase. A suitable compound must be able to exist in a nonionic form in the donor phase and in ionic form in the acceptor phase. Due to the difference in concentration and $\mathrm{pH}$ 
between the phases, the compound of interest is irreversibly driven through the oil phase by diffusion and is enriched in the acceptor phase, reaching much higher concentrations than in the donor. High selectivity and enrichment factors can be achieved through accurate choice of the membrane and oil phase, and by tuning the $\mathrm{pH}$ and flow rates of the donor phase.

There are numerous studies focused on the development of sample preparation and extraction techniques in combination with SERS detection, especially when dealing with complex sample matrices. ${ }^{22,23}$ In our previous work, ${ }^{23}$ we demonstrated the advantages of combining traditional LLE with SERS detection and successfully screened different $E$. coli strains based on the produced pHCA quantity. However, LLE proved to have some disadvantages, since it required several manual sample handling steps and the usage of toxic solvents.

SLM extraction represents an attractive tool for sample microfluidic SLM device and quantified with SERS. The method was validated using HPLC.

\section{Experimental}

\section{Chemicals}

$100 \mathrm{mM}$ pHCA and $5 \mathrm{mM}$ Tyr stock solutions were freshly prepared in ethanol (EtOH) $99 \%$ and in $100 \mathrm{mM} \mathrm{NaOH}$ respectively. Dihexyl ether (DE) $97 \%$ was used for wetting the membranes in the SLM extraction devices. Donor samples were acidified at $\mathrm{pH} 1$ with $\mathrm{H}_{2} \mathrm{SO}_{4} 0.5 \mathrm{M}(10 \% \mathrm{v} / \mathrm{v})$, whereas 10 $\mathrm{mM}$ phosphate buffer (PB), $\mathrm{pH} 7.4$, was used as acceptor buffer for SLM extraction. For pHCA production assays the $E$. coli cells were grown in minimal M9 medium, ${ }^{27}$ containing 10 $\mathrm{g} / \mathrm{L}$ glucose, $2 \mathrm{mM}$ Tyr, $1 \mathrm{mM}$ IPTG and Wolfe's Vitamin solution purchased from ATCC $^{\circledR}$ (LGC Standards, UK) as extensively described by Morelli et al. ${ }^{23}$ Aqueous solutions
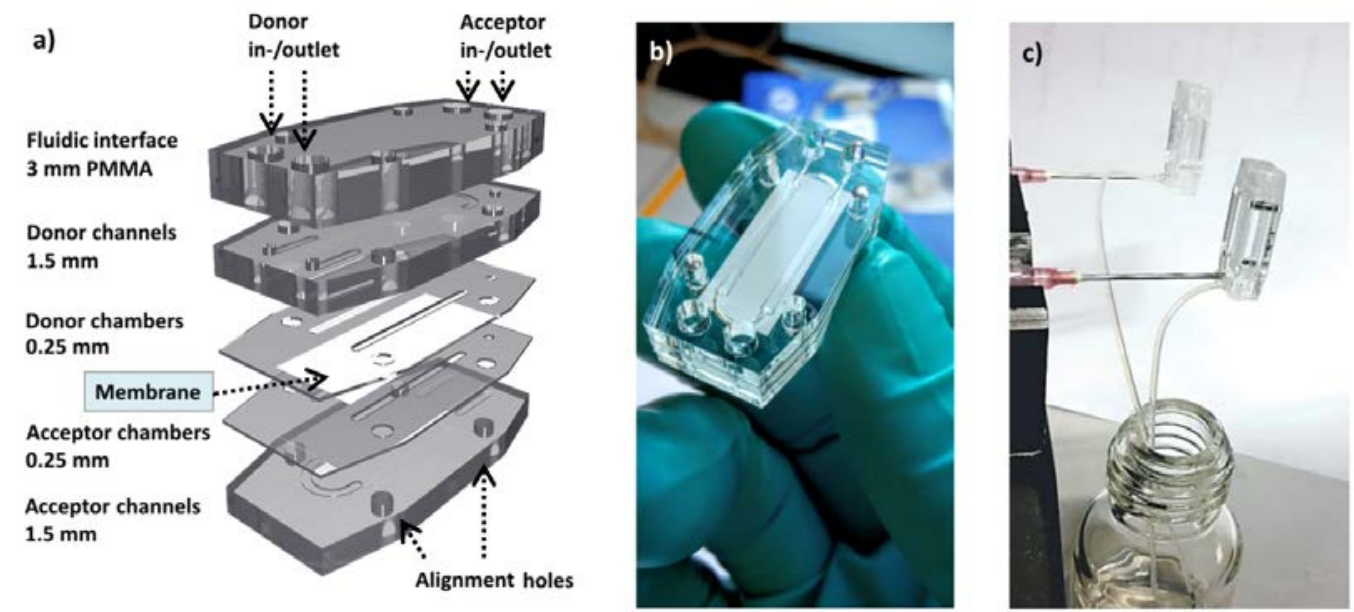

Fig. 1: (a) Exploded view of the different layers of the SLM extraction device. (b) Fluidic unit after thermal bonding, before inserting the silicone connectors. (c) Two extractions running in parallel, using a dual syringe pump and a common waste.

pretreatment. ${ }^{20}$ It is environmentally friendly, as low volumes of organic solvents are used, ${ }^{24}$ selective, ${ }^{25}$ able to reach high enrichment factors, ${ }^{26}$ tunable for specific applications, easy to implement and shows high potential for automation. Although there are many examples in literature on integration of SLM extraction modules with conventional detection techniques, such as liquid or gas chromatography and capillary electrophoresis, ${ }^{26}$ to the best of our knowledge it was not used in combination with SERS-based detection. Given the challenges in SERS-based sensing, especially when dealing with water-based complex matrices, the selectivity of extraction and enrichment enabled by SLM extraction are of particular interest.

In this work we coupled SLM extraction to SERS-based detection and demonstrated the effectiveness of the method through a rapid screening of $E$. coli strains cultivated in different medium compositions. The metabolite of interest ( $\mathrm{pHCA})$ produced by each strain was extracted using a were made with ultrapure water obtained from a Milli-Q purification system (Millipore Corporation, Billerica, MA, U.S.). All the chemicals were purchased from Sigma-Aldrich, unless otherwise stated.

\section{E. coli culture}

E. coli control and PHCA producing strains were constructed as described by Jendresen et al., ${ }^{7}$ from the expression strain BL21(DE3)pLysS (Invitrogen/Life Technologies), carrying the extrachromosomal cloning vector pCDFDuet-1 or a derived plasmid encoding the tyrosine ammonia-lyase FjTAL. The pHCA producing strain was grown with $2 \mathrm{mM}$ Tyr in different medium compositions (complete M9 medium, ${ }^{23}$ absence of IPTG or absence of vitamins). The control strain, characterized by the absence of FjTAL, was grown without Tyr and IPTG in M9 medium. Samples were taken at $24 \mathrm{~h}$, and supernatant was obtained by double centrifugation (10 $\mathrm{min}$ at $\left.10000 \mathrm{~g}, 4^{\circ} \mathrm{C}\right)$.

\section{Microfluidic SLM device and experimental setup}


The microfluidic SLM extraction units were designed using CAD software (AutoCAD, Autodesk Inc., San Rafael, CA, US). As presented in Fig. 1(a), the fluidic system was made of layers of polymethyl methacrylate ((PMMA, Goodfellow Cambridge Ltd, Huntingdon, England)) with different thickness (from 0.25 to 3 $\mathrm{mm}$ ) using laser ablation (Epilog Mini 18, 30W from Epilog Laser, Golden, CO, US). For assembly the PMMA layers were rinsed with isopropanol followed by 2 minutes of UV exposure (DYMAX 5000 EC, bulb 36970 (Dymax Corp., CT, U.S.)), aligned and bonded together via thermal bonding $\left(85^{\circ} \mathrm{C}, 25\right.$ bar for 40 minutes) in a bonding press (PW $10 \mathrm{H}, \mathrm{P} / \mathrm{O} /$ Weber, Germany) (Fig. 1(b)). The nanoporous polypropylene (PP) membrane (Celgard 2500 Membrane, 55\% porosity, $64 \mathrm{~nm}$ average diameter pore size and $25 \mu \mathrm{m}$ thick, Celgard, Charlotte, NC, U.S.) was cut into $12 \times 24 \mathrm{~mm}$ pieces and integrated in the system during the thermal bonding procedure (Fig. 1(a)). The fluidic system was designed with two extraction chambers ( $1 \mathrm{x}$ $0.25 \times 35 \mathrm{~mm}$ ) connected in series by a U-shaped channel (Fig. 2), for a total volume of $15 \mu \mathrm{L}$ for each phase with inlet and outlet placed on the same side of the microfluidic chip for both donor and acceptor. Small pieces of silicone tubes $(3 \mathrm{~mm}$ outer diameter, $1 \mathrm{~mm}$ inner diameter, $3 \mathrm{~mm}$ length) were used as leak proof connectors at the inlets and outlets of the chip. The connectors were changed after each sample in order to avoid a possible cross-contamination between different samples.
The microfluidic device was prepared for extraction, by filling up the acceptor channel with PB in order to minimize oil contamination, and DE was introduced in the donor channel, followed by $15 \mathrm{~min}$ waiting time to enable membrane impregnation. After saturating the membrane with $D E$, the excess was removed and the donor channel was flushed with deionized water at $100 \mu \mathrm{L} / \mathrm{min}$ for 5 minutes. Before extraction, the acceptor channel was filled with $15 \mu \mathrm{L}$ PB and sealed with Parafilm $M^{\circledR}$ (Bemis Company, Inc., Neenah, WI, U.S.) and the device was connected to a syringe pump (Standard Infuse/Withdraw PHD 2000 Syringe Pump, Harvard Apparatus, Holliston, MA, U.S.) and to the waste as presented in Fig. 1(c). The samples were acidified $(\mathrm{pH} 1)$ in order to bring the analyte ( $\mathrm{pHCA})$ in its neutral form and enable its diffusion through the SLM into the acceptor phase. $\mathrm{A} \mathrm{pH}$ of 7.4 in the acceptor phase protonates pHCA, which cannot diffuse back through the SLM to the donor phase (Fig. 2 (inset)).

After the extraction, the donor channel was filled with $50 \mathrm{mM}$ $\mathrm{H}_{2} \mathrm{SO}_{4}$ and left to equilibrate for $10 \mathrm{~min}$. The acceptor was removed and diluted with EtOH for SERS detection as schematically presented on Fig. 2. Finally the acceptor channel was rinsed with $\mathrm{PB}$ at $100 \mu \mathrm{L} / \mathrm{min}$ for $5 \mathrm{~min}$.

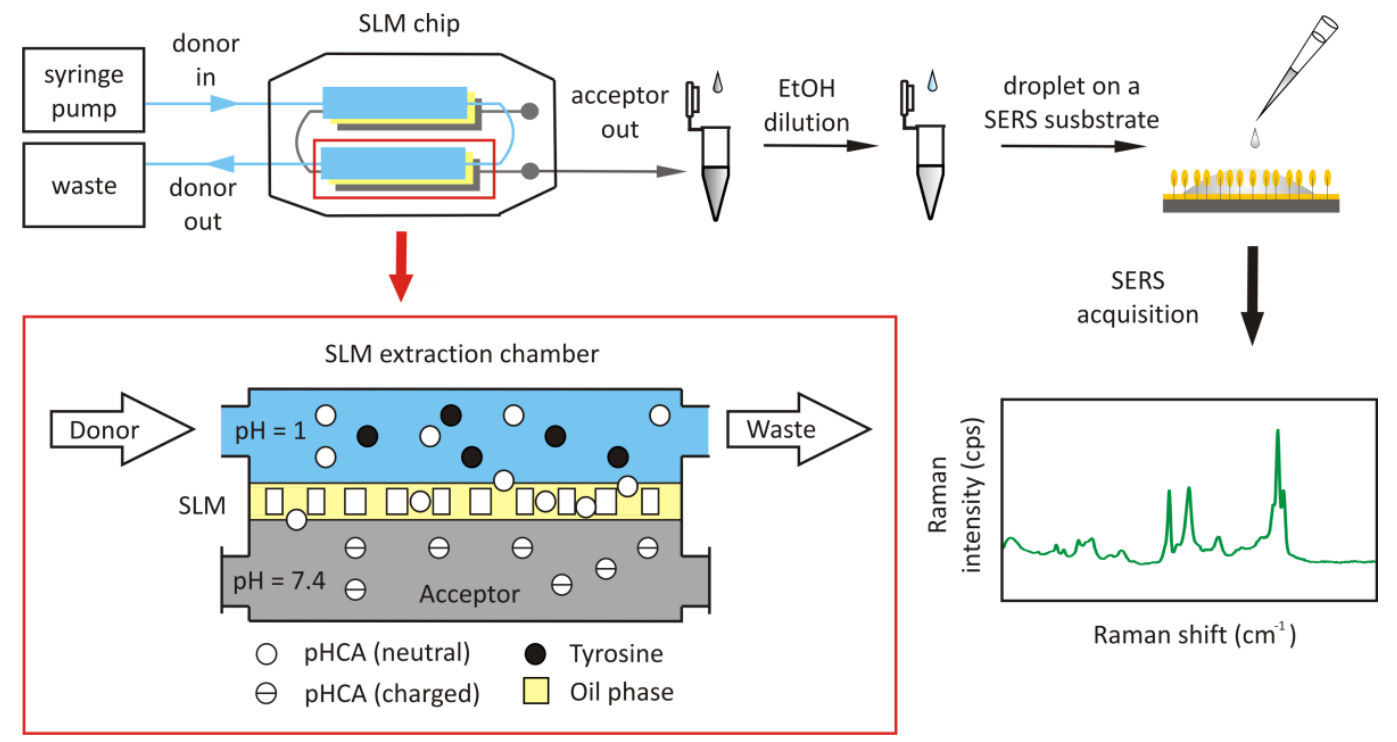

Fig. 2: Experimental setup for SLM extraction and SERS analysis. From left to right, a syringe pump is connected to the donor compartment of the SLM chip, and the acceptor compartment is filled with PB. After extraction the acceptor is taken and diluted 10 times with EtOH 99\%. $2 \mu \mathrm{L}$ droplet of the diluted extract is poured and dried on a $4 \times 4 \mathrm{~mm}^{2}$ SERS chip, followed by Raman acquisition. (inset) SLM working principle. 


\section{SERS chip fabrication and data acquisition}

The gold-capped nanopillars substrates were fabricated with the methods described by Wu et al., ${ }^{28}$ with 4 min etching time, followed by $1 \mathrm{~min} \mathrm{O}_{2}$ plasma cleaning, deposition of $220 \mathrm{~nm}$ $\mathrm{Au}$ at a rate of $10 \AA \mathrm{A} / \mathrm{s}$ and dicing of $4 \times 4 \mathrm{~mm}^{2}$ substrates. The acceptor samples were taken after SLM extraction and diluted 10 times with $\mathrm{EtOH} 99 \%$, in order to increase the spreading of the droplet on the SERS substrates. Each substrate was wetted with a $2 \mu \mathrm{L}$ droplet, and dried completely before acquisition. SERS measurements were performed with a DXRxi Raman Imaging Microscope (Thermo Fisher Scientific Inc., Waltham, MA, U.S.). The optical microscope is coupled to a spectrometer $5 \mathrm{~cm}^{-1} \mathrm{FWHM}$ and $\pm 2 \mathrm{~cm}^{-1}$ wavenumber accuracy. SERS spectra were collected at $780 \mathrm{~nm}$ with a laser power of $1 \mathrm{~mW}$, with a 10x objective lens, $50 \mu \mathrm{m}$ slit and an estimated laser spot of $3.6 \mu \mathrm{m}$ diameter. All the spectra were collected 3 times for 0.05 s. 3 maps of 48 spectra each were collected on the surface of each chip, with a $100 \mu \mathrm{m}$ collection step. The peak height at $1169 \mathrm{~cm}^{-1}$ was used for quantification, according to the methods previously described by Morelli et al. ${ }^{23}$ The average Raman intensity and the corresponding standard deviation for each sample were calculated over the average values of the maps collected on the same chip.

\section{HPLC acquisition}

Supernatant and acceptor samples were diluted 10 times with ultrapure water. Triplicate injections $(5 \mu \mathrm{L})$ were analyzed at $30{ }^{\circ} \mathrm{C}$ in an HPLC system (Thermo Scientific, Waltham, MA, US) with a Discovery HS F5 column ( $3 \mu \mathrm{m}$ particle size, $15 \mathrm{~cm} \times 4.6$ $\mathrm{mm}$ ). Elution was performed using a gradient with two solvents: $10 \mathrm{mM}$ ammonium formate adjusted to $\mathrm{pH} 3.0$ with formic acid (A) and acetonitrile (B) running at $0.7 \mathrm{~mL} / \mathrm{min}$, starting at $5 \% \mathrm{~B}$. The fraction of $\mathrm{B}$ increased linearly from $5 \%$ to $60 \%$ from $1.5 \mathrm{~min}$ to $7 \mathrm{~min}$ after injection. Then the fraction of $B$ decreased back to $5 \%$ between 9.5 and $9.6 \mathrm{~min}$, and remained there until $12 \mathrm{~min}$. pHCA was detected by absorbance at $333 \mathrm{~nm}$.

\section{Results and discussion}

\section{SERS-based detection and quantification of pHCA}

Measuring analytes in aqueous samples is not ideal in SERSbased sensing due to low surface wettability. In addition, based on our previous finding we observed that salts from growth medium decrease the signal intensity, ${ }^{18}$ therefore, we constructed pHCA calibration curves using different ratios of $\mathrm{PB}$ and $\mathrm{EtOH}$. When using only $10 \% \mathrm{EtOH}$ we were not able to collect any SERS signal, due to insufficient surface wettability, which let the droplet stay round on the active surface, and to subsequent salt accumulation. Instead, when using 50 or $90 \%$ EtOH, we successfully measured pHCA (Fig. S1). Due to faster evaporation, $90 \%$ EtOH dilution was chosen for all the experiments. The typical spectrum of pHCA is shown in Fig. $3(a)$, with peak assignment described previously, ${ }^{23}$ and the calibration based on Raman intensity at $1169 \mathrm{~cm}^{-1}$ is shown in Fig. 3(b). SERS substrates showed a linear response up to 250
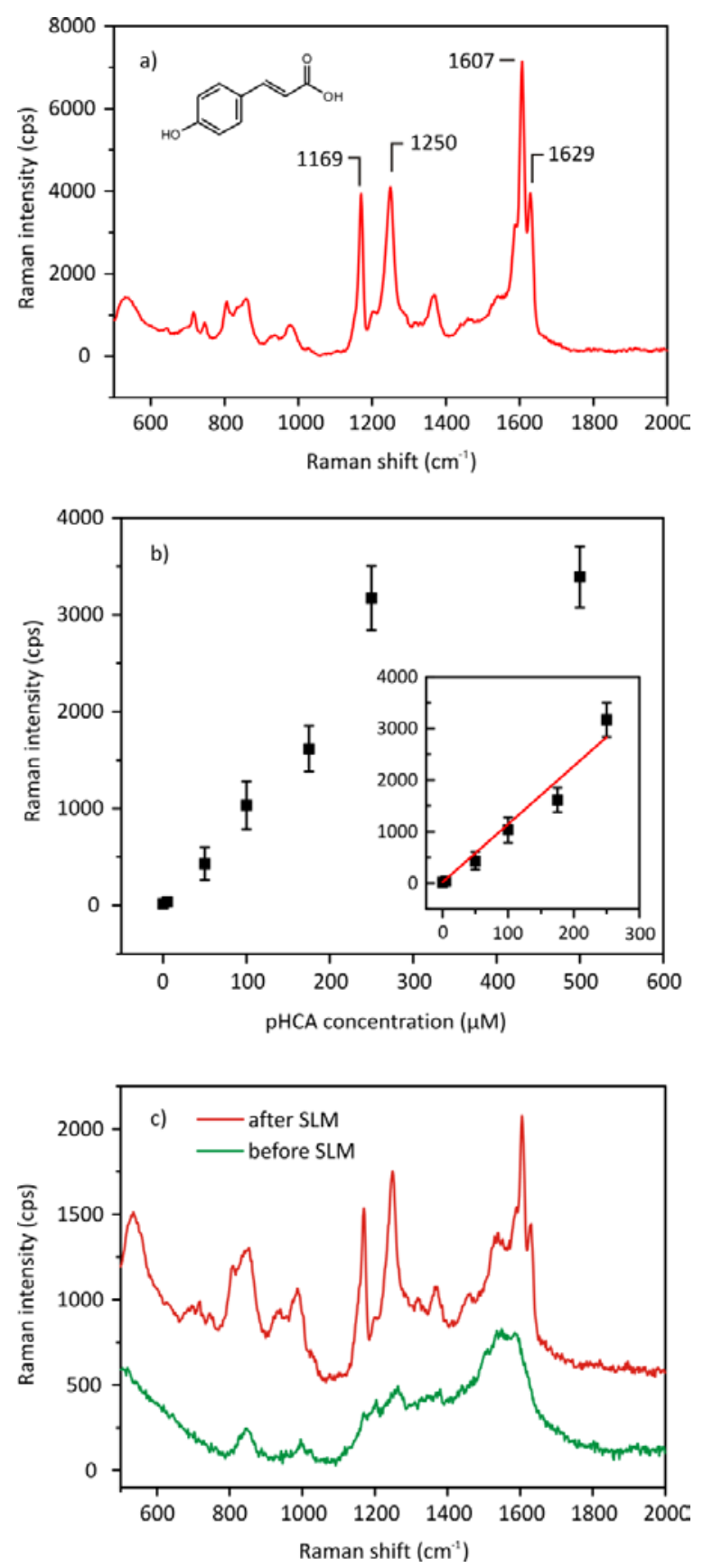

Fig. 3: (a) Typical SERS signal of pHCA. The spectrum is the average of 48 points, acquired on the surface of a chip wetted with $2 \mu \mathrm{L}$ of $250 \mu \mathrm{M}$ pHCA in EtOH/PB $90 \%$ v/v. (b) calibration curve obtained by plotting Raman intensity at $1169 \mathrm{~cm}^{-1}$ versus pHCA concentration in EtOH/PB $90 \% \mathrm{v} / \mathrm{v}$. The data in the graph is the combination of data acquired in 3 independent experiments, each one performed at least in duplicate chips, with error bars representing the overall standard deviation. (c) Comparison between SERS spectra of M9 medium spiked with $250 \mu \mathrm{M}$ pHCA and $750 \mu \mathrm{M}$ Tyr before and after SLM extraction, diluted 10-fold with EtOH. Each spectrum was obtained as the average of 48 points, collected on the surface of a chip wetted with a $2 \mu \mathrm{L}$ droplet.

$\mu \mathrm{M}$, which was used for quantification of unknown samples (Fig. 3(b), inset). Limit of detection and limit of quantification 
were found to be 5 and $15 \mu \mathrm{M}$ respectively, whereas the average coefficient of variation, calculated in a concentration range between 50 and $250 \mu \mathrm{M}$, was found to be $22 \%$. Fig. 3(c) shows a comparison between SERS spectra of M9 medium spiked with $250 \mu \mathrm{M}$ pHCA and $750 \mu \mathrm{M}$ Tyr before and after SLM extraction, diluted 10 -fold with $\mathrm{EtOH}$, in order to remove the effect of salts. It can be seen that SLM extraction significantly enhances pHCA signal, while excluding Tyr overlapping spectral features ${ }^{23}$ (Fig. S2), hence proving effective for removal of interfering compounds.

\section{Reusability of the SLM microfluidic chip}

In order to study the reusability and the possibility of cleaning the SLM microfluidic unit, supernatant samples containing 450 $\mu \mathrm{M}$ pHCA were extracted at $100 \mu \mathrm{L} / \mathrm{min}$ using the SLM device. Several cycles of extraction and rinsing were performed on the same microfluidic unit in order to evaluate and determine the efficiency of the cleaning as well as the reproducibility of the SLM extraction. After each extraction and rinsing step the acceptor was recovered and measured with SERS following the protocol depicted in Fig. 2. The normalized values are presented in Fig. 4, where $100 \%$ represents pHCA concentration obtained in the first SLM extraction. The extraction proved to be reproducible even when performing several cycles of extraction and rinsing. The memory effect was further investigated using different rinsing methods under flow condition (dynamic rinsing), as described in the experimental section, and static condition (static rinsing), by filling the acceptor channel with PB and letting sit for $10 \mathrm{~min}$. The signal decreased from $4 \%$ to $2 \%$ when performing static rinsing three times, whereas it reached less than $3 \%$ already after a single dynamic rinsing, showing that the SLM device could be efficiently cleaned under flow condition (Fig. S3). Based on these observations, a single dynamic rinsing was always performed when reusing chips for experiments.

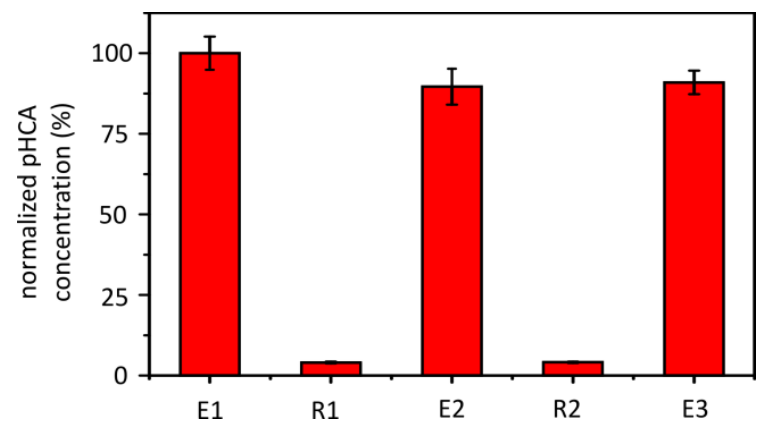

Fig. 4: Subsequent SLM extractions (E1, E2, E3) and dynamic rinsing $(R 1, R 2)$ performed on the same microfluidic chip. The values represent the normalized pHCA concentration from SERS acquisitions, with error bars representing the standard deviation of 3 maps acquired on a chip. The concentration is normalized versus the concentration obtained in the first SLM extraction.

\section{Effect of the donor flow rate on analyte enrichment}

As defined by Jönsson et al. ${ }^{26}$ the extraction efficiency (E) can be described as:

$$
E=\frac{n_{A}}{n_{D}}
$$

where $n_{A}$ and $n_{D}$ are the number of moles collected in the acceptor and given as input to the system through the donor respectively. The enrichment factor $\left(E_{e}\right)$ can then be defined as:

$$
E_{e}=E \cdot \frac{V_{D}}{V_{A}}=\frac{E \cdot F_{D} \cdot t}{V_{A}}
$$

where $V_{D}$ is the donor volume, $V_{A}$ is the acceptor volume, $F_{D}$ is the volumetric flow rate of the donor phase, and $t$ is the total time that donor is in contact with the membrane.

To be able to define the optimum extraction condition,
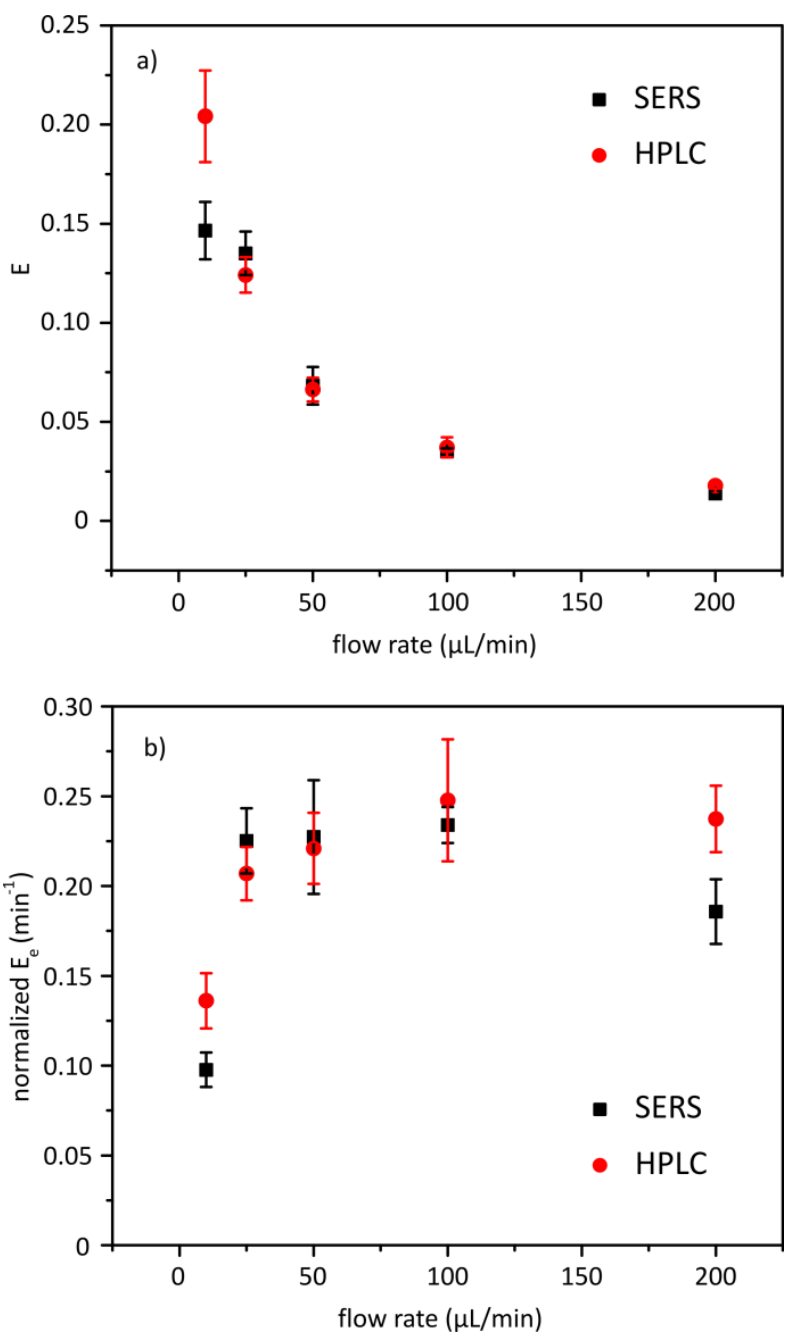

Fig. 5: (a) $E$ and (b) time-normalized $E_{e}$ versus flow rate based on data from HPLC and SERS. Each extraction was performed at least in triplicates with different SLM devices, and each extract was tested on a SERS chip and with triplicate HPLC injections. The error bars represent the overall standard deviation in both cases.

extractions were performed on samples spiked with $250 \mu \mathrm{M}$ pHCA and $750 \mu \mathrm{M}$ Tyr at different flow rates. A fixed volume of $1 \mathrm{~mL}$ acidified sample was passed through the SLM unit at 10, 
$25,50,100$ and $200 \mu \mathrm{L} / \mathrm{min}$ respectively. pHCA concentration in the recovered acceptor extracts was analyzed with SERS and HPLC.

As expected from theory, ${ }^{26}$ we found that $E$ increases with decreasing flow rate (Fig. 5(a)), from $0.02 \pm 0.001$ at 200 $\mu \mathrm{L} / \mathrm{min}$ to $0.20 \pm 0.02$ at $10 \mu \mathrm{L} / \mathrm{min}$. Considering the $\mathrm{pHCA}$ content in the donor phase, we found that its concentration in the acceptor phase increased, reaching an up-concentration of 13.6-fold when decreasing the flow rate to $10 \mu \mathrm{L} / \mathrm{min}$. In order to evaluate the effect of flow rates at a fixed sample volume, we normalized $E_{e}$ by extraction time. As it can be seen in Fig. $5(b)$, the normalized $E_{e}$ increases with increasing flow rate until $100 \mu \mathrm{L} / \mathrm{min}$.

Considering the linear part of the SERS calibration curve (Fig $3(b)$, inset) and the time required by the SLM extraction using $1 \mathrm{~mL}$ of sample, $100 \mu \mathrm{L} / \mathrm{min}$ flow rate (10 min of extraction) was chosen for the experiments.

\section{Quantification of pHCA in bacterial supernatant}

In order to assess the applicability of the method for screening different culture conditions, SLM extraction was performed on bacterial supernatant samples. As described in the Materials and Methods section, an E. coli strain expressing a tyrosine ammonia-lyase (FjTAL) was grown in different medium compositions (complete M9, absence of IPTG and absence of vitamins). A control strain, characterized by the absence of FjTAL, was also tested. pHCA concentrations in the extracts were measured with SERS and compared to the concentrations measured in the supernatant with HPLC, considering an $E_{e}$ of

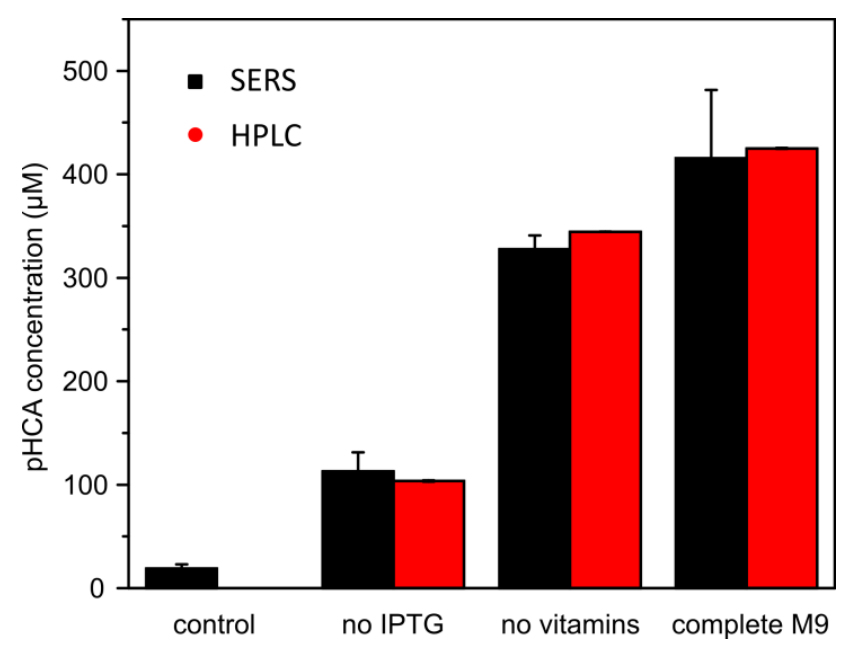

Fig. 6: Comparison between HPLC measurements of pHCA concentration in bacterial supernatant and SERS measurements after SLM extraction. Extractions were performed at least in triplicates with different SLM devices, each extraction being tested on a SERS chip. HPLC data were obtained analysing bacterial supernatant with triplicate injections. Error bars represent overall standard deviation in both cases.

0.23 at $100 \mu \mathrm{L} / \mathrm{min}$. pHCA quantification in Fig. 6 highlights a leaky expression of FjTAL in absence of IPTG, and a reduced pHCA production in absence of vitamins, compared to complete M9 medium. In all cases, a close correlation between

SERS and HPLC data could be observed, showing that quantitative differentiation between bacteria grown in different conditions can be successfully performed through a combination of SLM extraction and SERS. Additionally, pHCA quantification was not affected by the presence of residual Tyr in supernatant, which was found to be $420 \mu \mathrm{M}$ in absence of IPTG, $235 \mu \mathrm{M}$ in absence of vitamins and $82 \mu \mathrm{M}$ in complete M9.

\section{Conclusions}

In this work we proved the usability and the applicability of SLM extraction for sample pretreatment prior to SERS sensing. We demonstrated that the combination of SLM extraction and SERS can be effectively used as an alternative to standard analytical methods for screening of $E$. coli strains based on their pHCA synthesis yield. The SLM microfluidic device has significant advantages compared to our previously developed manual LLE assay, ${ }^{23}$ being easy to handle and using only a few microliters of organic solvent for membrane impregnation. The device proved solid and effective for extraction and upconcentration of pHCA from real samples and its reusability significantly increased the throughput of the assay. The data obtained from bacterial supernatant with SLM extraction coupled with SERS detection showed a good correlation with HPLC analysis, proving the value of the method for quantitative screening of genetically modified organisms.

The great potential for automation and miniaturization of the presented method widens its applicability in a number of different fields. For instance, a miniaturized SLM/SERS device could be easily adapted for automated and/or real time monitoring, making it attractive for various applications such as detection of other industrially relevant bacterial metabolites, toxins and pharmaceutical compounds.

\section{Conflicts of interest}

There are no conflicts of interest to declare.

\section{Acknowledgements}

The research was funded by Danish National Research Foundation (DNRF122), Villum Fonden (Grant No. 9301), the European Research Council (320535, 'HERMES'). Christian Bille Jendresen was supported by The Novo Nordisk Foundation (Grant No. NNF15OC0015246).

\section{References}

\author{
J. A. Dietrich, A. E. McKee and J. D. Keasling, Annu. Rev. \\ Biochem., 2010, 79, 563-590. \\ J. Nielsen and J. D. Keasling, Nat. Biotechnol., 2011, 29, \\ 693-695. \\ J. K. Rogers and G. M. Church, Trends Biotechnol., 2016, 34, \\ 198-206. \\ P. Jacques, M. Béchet, M. Bigan, D. Caly, G. Chataigné, F.
}


Coutte, C. Flahaut, E. Heuson, V. Leclère, D. Lecouturier, V. Phalip, R. Ravallec, P. Dhulster and R. Froidevaux, Bioprocess Biosyst. Eng., 2017, 40, 161-180. H. M. Hegab, A. EIMekawy and T. Stakenborg, Biomicrofluidics, 2013, 7, 21502. J. W. Lim, K. S. Shin, J. Moon, S. K. Lee and T. Kim, Anal. Chem., 2016, 88, 5234-5242. C. B. Jendresen, S. G. Stahlhut, M. Li, P. Gaspar, S. Siedler, J. Förster, J. Maury, I. Borodina and A. T. Nielsen, Appl. Environ. Microbiol., 2015, 81, 4458-4476. A. Ribeiro, M. Estanqueiro, M. Oliveira and J. Sousa Lobo, Cosmetics, 2015, 2, 48-65. N. Balasundram, K. Sundram and S. Samman, Food Chem., 2006, 99, 191-203. J. A. Jones, S. M. Collins, V. R. Vernacchio, D. M. Lachance and M. A. G. Koffas, Biotechnol. Prog., 2016, 32, 21-25. H. S. Kim, T. L. Weiss, H. R. Thapa, T. P. Devarenne and A. Han, Lab Chip, 2014, 14, 1415-25. Y. Tang, M. Gan, Y. Xie, X. Li and L. Chen, Lab Chip, 2014, 14, 1162-7.

13 P. L. Stiles, J. A. Dieringer, N. C. Shah and R. P. Van Duyne, Annu. Rev. Anal. Chem., 2008, 1, 601-626.

14 M. Prochazka, in Surface-Enhanced Raman Spectroscopy: Bioanalytical, Biomolecular and Medical Applications, Springer International Publishing, Cham, 2016, pp. 61-91. R. K. Lauridsen, T. Rindzevicius, S. Molin, H. K. Johansen, R. W. Berg, T. S. Alstrøm, K. Almdal, F. Larsen, M. S. Schmidt and A. Boisen, Sens. Bio-Sensing Res., 2015, 5, 84-89. S. Nie, Science, 1997, 275, 1102-1106.

E. Petryayeva and U. J. Krull, Anal. Chim. Acta, 2011, 706, 8-24.

18 L. Morelli, C. B. Jendresen, K. Zor, T. Rindzevicius, M. S. Schmidt, A. T. Nielsen and A. Boisen, Procedia Technol., 2017, 27, 190-192.

19 M. Robert, D. Murray, M. Honma, K. Nakahigashi, T. Soga and M. Tomita, 2012 ICME Int. Conf. Complex Med. Eng. C. 2012 Proc., 2012, 197-202.

20 L. Chimuka, E. Cukrowska and J. Å. Jönsson, Pure Appl. Chem., 2004, 76, 707-722.

21 J. Å. Jönsson and L. Mathiasson, J. Chromatogr. A, 2000, 902, 205-225. A. Kim, S. J. Barcelo, R. S. Williams and Z. Li, Anal. Chem., 2012, 84, 9303-9.

23 L. Morelli, K. Zór, C. B. Jendresen, T. Rindzevicius, M. S. Schmidt, A. T. Nielsen and A. Boisen, Anal. Chem., 2017, 89, 3981-3987. J. A. Jönsson and L. Mathiasson, TrAC Trends Anal. Chem. 1999, 18, 318-325. B. Lindegård, H. Bjork, J. Å. Jonsson, L. Mathiasson and A. M. Olsson, 1994, 66, 4490-4497.

26 J. Å. Jönsson and L. Mathiasson, TrAC Trends Anal. Chem., 1999, 18, 325-334.

27 J. H. Miller, Experiments in Molecular Genetics, Cold Spring Harbor Laboratory, 1972.

28 K. Wu, T. Rindzevicius, M. S. Schmidt, K. B. Mogensen, A. Hakonen and A. Boisen, J. Phys. Chem. C, 2015, 119, 20532062. 